\title{
PROGRESS TOWARD AN IMPROVED EQUATION OF STATE AND OPACITY FOR STELLAR ENVELOPES
}

\author{
Dimitri Mihalas \\ Department of Astronomy, University of Illinois
}

\begin{abstract}
A brief description is given of a large international project for computing new stellar envelope opacities and equation of state data.
\end{abstract}

INTRODUCTION

For the past four to five years a large project to recompute opacities for stellar envelopes has been underway. Approximately 20 physicists and astrophysicists are contributing to this effort, the largest group being located in the UK (under the leadership of M.J. Seaton and P.G. Burke), a much smaller group in the US, and a few individuals in other countries. The non-us team members are responsible primarily for producing the requisite atomic data, while the us group has been working on the equation of state (EOS), and on combining cross-sections with the EOS to produce opacities.

For over 25 years the astrophysical community has relied almost exclusively on opacity calculations done at Los Alamos, and it is natural to ask "Why should this job be done over?". There are a number of partial answers to this question. First, we all have simply accepted the Los Alamos results without (because the codes and databases are not in the public domain) being able to evaluate them critically. Yet we know that many phases of stellar evolution depend sensitively on the opacity, and so long as we have only one set of data available to us, we cannot objectively assess the implications of alternative methods of calculating the opacity for computations of stellar structure, evolution, and pulsation. Second, there have long been some annoying discrepancies in Cepheid models and observations, and the existing evidence points towards the need for a significant increase in the opacity in regions of the $(\boldsymbol{\rho}, \mathrm{T})$ plane near where $\mathrm{He}, \mathrm{C}, \mathrm{N}$, and $\mathrm{O}$ are ionizing. Third, it has been only very recently that numerical methods for calculating atomic structure and the optical properties of atoms have been able to produce these data quickly. As a result, only now is it possible to produce an essentially complete set of atomic data of very high quality. Fourth, the computers are now large enough and fast enough to permit us to resolve the spectrum, including lines, over the entire Rosseland-mean window. Finally, if we succeed with this project, astronomers will have, for the first time, a facility (hardware + software + documentation + data) that will allow them to carry out the calculations themselves for any mix they desire. 
THE ATOMIC DATA

Wave functions and energy levels are being computed by the R-matrix method for all ions of all astrophysically abundant elements up through $\mathrm{Fe}$. At present all elements up through $\mathrm{Ne}$ are complete, and work on third period elements (up through Ar) is underway. Initial studies on $\mathrm{Fe}$ have begun. These calculations give a complete set of oscillator strengths for all lines through upper principal quantum number $=10$; beyond that we use hydrogenic values. A characteristic of this work is that continuum cross-sections are typically riddled with sharp resonances. As a result, the distributions of differential oscillator strength is not even roughly hydrogenic; this one fact could lead to large changes from presently accepted values of opacities.

When these computations are completed, we shall have an atomic dataset unprecedented in both quality and extent. However we must also point out a basic limitation of our approach: all of the atomic data apply, strictly speaking, only to isolated atoms, i.e. atoms (and ions) whose internal structure is not significantly altered by the presence of the other particles in the plasma. As a result, we cannot realistically treat high-density material, such as exists in stellar cores. In practice we have chosen an upper density bound of 0.01 grams per cubic centimeter; this limit is quite adequate for treating, e.g. the pulsational properties of Cepheid and RR Lyrae envelopes.

\section{EQUATION OF STATE}

Our equation of state is based on a free-energy minimization method. The free energy includes contributions from (1) the translational motions of classical nuclei; (2) the internal excitation states of molecules, atoms, and ions; (3) the translational motions of semi-degenerate electrons; and (4) Coulomb interactions among all charged particles.

Our implementation of this method differs from others like it in the literature in two important respects. First, we allow each atom or ion to have many hundreds of bound states (the uppermost of which are hydrogenic). We have used all available empirical energy levels, and supplemented them where necessary with values obtained by extrapolating quantum defects up series. Then in order to truncate the internal partition function we introduce occupation probabilities $w(i)$ which depends on the binding energy $E(i)$ of state $i$ and a weighted sum of ionic charges times the numbers of corresponding ions. To calculate $w(i), D$. G. Hummer made a thorough quantum mechanical investigation of the rate of Stark ionization of a bound electron by an imposed fluctuating field. The form of $w(i)$ is such that $w$ approaches unity for strongly bound (i.e. low-lying) states, and w approaches zero for weakly bound states lying close to the ionization continuum. The effective quantum number at which $w$ makes the transition from near unity to near zero is a monotonically decreasing function of the ion charge density. 
Second, because $w(i)$ is chosen to be continuous (indeed differentiable to all orders) atomic states do not vanish abruptly as they do in other formulations, hence discontinuities in the free energy (which would produce delta functions in the pressure and/or internal energy) are avoided. In fact, all four contributions to the free energy are analytical and differentiable. As a result it is possible to compute all secondary thermodynamic properties (specific heats, adiabatic gammas, etc.) analytically, without numerical differentiation. The evaluation of these formulae, though laborious, yields very smooth values, and provides a powerful numerical check on the whole calculation.

At present we have calculated EOS tables for 6 mixtures of 15 elements from $\mathrm{H}$ through $\mathrm{Fe}$; in all 205 distinct species of particles (including hydrogen molecules, hydrogen molecule ions, negative hydrogen ions, and free electrons) are considered. We have chosen 6 representative mixtures ranging from super-metal-rich $([\mathrm{Fe} / \mathrm{H}]=+0.5)$, through solar, to extremely metal poor $([\mathrm{Fe} / \mathrm{H}]=-2)$. We have been able to check some of our results against Los Alamos computations. In general the agreement is good, though, as noted by Pesnell, the hydrogen ionization loci in the temperature-density plane computed from the two theories do not coincide. This discrepancy is a direct result of the two different methods of handling the internal partition function, and suggests that there could be significant differences in our opacity values, compared to Los Alamos, near ionization loci.

We are now prepared to release EOS data to "friendly users". They can be obtained by sending a tape to D. Mihalas for a UNIX "tar" format or to D. G. Hummer for a blocked ASCII format (e.g. for VMS VAX). The UNIX tapes can be written on either standard $1 / 2^{n}$ tape or a $1 / 4^{n}$ tape cartridge (preferred); ASCII tapes must be $1 / 2 \pi$. We hope that users will keep us informed of problems and errors they encounter.

\section{OPACITIES}

A first draft of the opacity code is virtually completed. Still needed is a quick way to compute stark profiles, and code to compute multigroup means and an archival set of opacity distribution functions. These tasks are being worked on now.

In our opacities we attempt to include effects of the occupation probabilities used in the EOS on the optical properties of the gas. These effects can be quite important, when, for example, a sequence of lines converging on an ionization limit is blurred into a pseudocontinuum as the upper states are destroyed, and what normally would be a bound-bound transition between states " 1 " and " $u$ " becomes a bound-free transition because the upper state no longer exists as a bound state. A similar effect by which bound-free transitions are converted into free-free also occurs. We expect the occupation probabilities will affect our opacities in certain regions of the temperature-density plane, a point that we shall study at a later date. 
At present we have carried out only a few experimental calculations for $\mathrm{H}, \mathrm{He}, \mathrm{C}$, and some mixes of these elements. We hope to add $\mathrm{N}$ and $\mathrm{O}$ by the end of the year. If all goes well we may be able to produce a table for, say, the solar mix by the end of 1989, though I suspect 1990 may be more realistic.

\section{ACKNOWLEDGEMENTS}

This research has been supported in part by grant number AST

85-19209 from the National Science Foundation to the University of

Illinois. I am pleased to be here representing my close coworkers Yu Yan, David Hummer, and Anil Pradhan, and all of our overseas colleagues (too numerous to list here). The cooperative spirit which has pervaded this project from its outset has made it much easier carry on in the face of the daunting realities of the work to be done. 